\title{
EBN perspectives: Care of the Older Person
}

\section{David Barrett ${ }^{1}$ and Laura Green ${ }^{2}$}

${ }^{1}$ Faculty of Health Sciences, University of Hull

${ }^{2}$ Division of Nursing, Midwifery and Social Work, University of Manchester

\section{Introduction}

This article is part of Evidence Based Nursing (EBN) Perspectives Series. In this series, published commentaries related to a specific nursing theme are collated and highlights are discussed. The topic for this edition is 'Older People', covering 20 commentaries published in 2017 and 2018. A summary of work is organized into key themes and important implications for practice and future research are explored.

\section{Key themes}

The 20 commentaries presented in Box 1 are grouped into four themes:

- Dementia care: Interventions that reduce the risk of dementia or improve the care of those people living with dementia.

- Falls and other injuries in older people: Identifying risk factors for falls and other injuries in older people, or exploring interventions to prevent them.

- Enhancing in-patient care for older people: Interventions to support the experience and outcomes of older people being nursed in hospital

- Lifestyle and health in older people: The impact of diet, exercise and other activities on wellbeing in older people

Box 1: Evidence-Based Nursing Commentaries on Care of the Older Person (20172018)

\section{Theme 1: Dementia care}

Pain related palliative care challenges in people with advanced dementia call for education and practice development in all care settings https://ebn.bmj.com/content/20/4/118

Evidence that active pain treatment improves sleep quality and quantity in people with depression and dementia. https://ebn.bmj.com/content/early/2018/12/18/ebnurs-2018102934 
Antihypertensive medications may reduce the risk of dementia in older African-Americans with hypertension https://ebn.bmj.com/content/early/2018/12/18/ebnurs-2018-102934

Better support and education is imperative to bolster informal at-home carers of people with cognitive impairment and reduce incidences of involuntary treatment of older adults in their homes. https://ebn.bmj.com/content/early/2019/01/04/ebnurs-2018-103028

Risk of injury higher in older adults with dementia than in those without https://ebn.bmj.com/content/20/4/117

\section{Theme 2: Falls and other injuries in older people}

Frailty significantly increases the risk of fractures among middle-aged and older people https://ebn.bmj.com/content/20/4/119

High-dose monthly vitamin D3 can help to prevent acute respiratory infections in older long-term care residents, but may increase risk of falls https://ebn.bmi.com/content/20/4/120

Antidepressant use associated with increased risk of hip fracture in the older population https://ebn.bmj.com/content/20/3/94

Exercise interventions reduce the risk of injurious falls among older adults https://ebn.bmj.com/content/21/3/75

Fear of falling-a hidden burden with or without a history of falls https://ebn.bmj.com/content/22/1/21

Evaluating falls prevention strategies in community settings: marginal reduction on rate of falls with individual risk based multifactorial interventions compared to 'usual care' https://ebn.bmi.com/content/22/1/20

\section{Theme 3: Enhancing in-patient care for older people}

Geriatric monitoring units could provide solution to ICU overcrowding and improve survival in older hospitalized patients https://ebn.bmj.com/content/20/3/93

The perspectives of patients and their families remain largely unheard in mealtime assistance research https://ebn.bmj.com/content/20/3/95

Commitment to patient-centred experiences at both the organization and clinician level optimises empowerment of hospitalized older adults with advanced disease https://ebn.bmj.com/content/21/1/27

Prehospital physical function and strength can influence clinical outcomes of critically ill older adults https://ebn.bmj.com/content/21/3/74

Inpatient mobilization programs targeting older patients are feasible but further research is required to examine impact on patient outcomes https://ebn.bmi.com/content/21/3/76

\section{Theme 4: Lifestyle and health in older people}

Engaging in even a small amount of walking is associated with lower mortality in older adults https://ebn.bmi.com/content/21/2/51 
Higher Vitamin $D$ intake could improve sleep and cognitive outcomes in older adults with heart failure https://ebn.bmj.com/content/21/3/69

Tai Chi: A promising adjunct nursing intervention to reduce risks of cardiovascular disease and improve psychosocial wellbeing in adults with hypertension.

https://ebn.bmi.com/content/early/2018/12/27/ebnurs-2018-103007

Effective dietary recommendations could help to prevent age-related cognitive decline https://ebn.bmj.com/content/21/1/26

\section{Implications for practice and future research}

The commentaries from the past two years reflect some of the most important issues related to older people and the care they receive. The first theme encompassed dementia, exploring interventions to reduce the risk of cognitive decline or improve the care of people living with dementia. Given the increasing prevalence of dementias, and the burden that they place on individuals and society, it is no surprise that there is such interest in this area of study.

Murray et al (2018) focused on interventions to reduce the risk of dementia in older people. Using a cohort study approach and recruiting African-Americans with hypertension, Murray and colleagues identified that those people prescribed antihypertensive medication were significantly less likely to develop dementia than those whose hypertension was left untreated.

Two studies recognised the importance of effective pain management in people living with dementia. Blytt et al (2018) explored the links between effective pain management and quality of sleep in people living with dementia. The randomised controlled trial demonstrated that active pain management can improve the quality and length of sleep, which it is known to have a beneficial impact on quality of life. De Witt et al (2017) adopted a qualitative approach to explore nurses' experiences of pain management for people with dementia who were approaching the end of life. Identifying a range of challenges that could impact on optimal pain management - including organisational barriers and professional relationships - the study highlighted the importance of opportunities for nurses to develop their skills and knowledge in this area of care.

There was some crossover between the quality of life of people living with dementia and the second theme - the prevention of falls or other injuries in older people. There is a wellevidenced link between dementia and falls, and this was highlighted by Meuleners \& Hobday (2017), who used a large-scale cohort study to identify that older people living with dementia 
had more than twice the risk of being admitted to hospital with an injury than those without dementia. Falls were the most common reason for injury-related hospitalisation, highlighting the importance of identifying and mitigating risk factors for falling in this group.

Three papers focused on specific factors that could increase the prevalence of falls - or worsen their consequences - in older people. Torvinen-Kiiskinen et al (2017) identified that the use of antidepressants was linked to a 2-3 times greater risk of hip fracture in older people, with the highest rate of injury amongst people with Alzheimer's disease. Other potential risk factors for falls and fractures identified within papers were frailty (Chen et al 2017) and - though it reduced the incidence of acute respiratory infections - the use of highdose monthly vitamin D (Ginde et al 2017)

It is crucial that nurses possess an understanding of interventions that can reduce the risk of falls, whatever their contributing factors. A systematic review by Tricco et al (2017) analysed randomised controlled trials of interventions designed to prevent falls. As a standalone intervention, exercise significantly reduced the incidence of falls, highlighting the importance of remaining active in older age. The study also demonstrated the importance and effectiveness of ensuring that the older person's environment is modified to reduce falls risk, and that steps are taken to optimise their visual acuity. This multifactorial approach to falls reduction was reinforced by Hoewell et al (2018), whose systematic review concluded that there was a reduction in the rate of falls associated with prevention strategies encompassing multiple interventions (e.g. exercise, medication optimisation and environmental enhancement). However, the authors did highlight that reductions were often marginal, and that the quality of evidence was often low-to-medium.

Theme three included a diverse range of commentaries that explored interventions to improve the care of older people in hospital. For some, these included a focus on vital elements of caring for older patients in hospital. For example, Edwards et al (2017) identified through a systematic review the importance of providing mealtime assistance to patients. Given the continuing problems associated with malnutrition in healthcare settings, the need to understand more about patients' experiences of mealtimes and how to enhance their nutritional intake remains a critical area of nursing research.

It is also crucial for nurses to understand other factors that influence the clinical outcomes of older adults in hospital. Files et al (2018) identified that amongst a cohort of older patients who had an Intensive Care Unit admission, lower levels of prehospital physical function (including walk speed and grip strength) were associated with greater risk of 30-day 
mortality. These findings reinforce the importance of nurses carrying out comprehensive, holistic patient assessments to identify high-risk older patients upon admission to critical care. It is also important to understand which interventions can be implemented to enhance clinical outcomes. One such intervention is the use of inpatient mobilisation programmes, offering a structured approach to increasing the activity levels of older inpatients. Liu et al (2018) demonstrated using a quasi-experimental approach (pre, during and postintervention) that such a programme could enhance levels of mobilisation and significantly reduce length of stay.

The final theme explored how lifestyle interventions, including diet and exercise, could enhance the health of older people. Theme four also included papers that demonstrated the benefits of specific interventions. For example, Chan et al (2018) demonstrated through a Randomised Controlled Trial that Tai Chi - a traditional Chinese exercise focused on mind and body - could improve psychosocial wellbeing and lower blood pressure more effectively than brisk walking alone.

Two large-scale longitudinal studies focused on the benefits of simple lifestyle interventions. Patel et al (2018) showed that engaging in walking as a form of physical activity in older age was associated with lower mortality rates and increased longevity, whilst McEvoy et al (2017) found that adherence to a neuroprotective diet (including the Mediterranean diet, which is rich in fruit and vegetables) had a beneficial impact on cognitive function in older adults.

Whilst the other three studies within this theme demonstrated the benefits of interventions the wider population, Song and Wu (2018) focused specifically on the needs of older adults with heart failure. Using a cross-sectional, observational approach, the study reinforced a growing evidence base that suggests increasing vitamin $D$ intake can be have benefits in heart failure patients related to sleep quality and cognitive function.

The recent commentaries in Evidence Based Nursing journal related to care of older people provide some important insights into factors which can influence and improve health and wellbeing. As the population ages, so it becomes increasingly important for nurses to understand the research evidence that identifies those factors that increase risk, and provides guidance on how best to support older people in mitigating these risks. This understanding should become embedded in all aspects of care, from patient assessment, through to communication, education and the implementation of evidence-based interventions that enhance, enrich and extend the lives of older people. 